\title{
Water Dynamics in Hydrated Carboxylated Cellulose
}

\section{Nanofibril Membranes}

Shun $\mathrm{Yu}^{1, *, \dagger}$, Valentina Guccini ${ }^{1}$, Franz Demmel $^{2}$, Germán Salazar-Alvarez $^{1, *, \sharp}$

1. Department of Materials and Environmental Chemistry (MMK), Stockholm University, SE10691, Stockholm, Sweden

2. ISIS Facility, Rutherford Appleton Laboratory, Didcot OX11 0QZ, UK

\begin{abstract}
:
Cellulose nanofibrils (CNF) are a class of materials with good mechanical properties, surface functionality and bio-/environmental friendliness. They have been used in many applications as loading material or function materials, where water-cellulose interaction determines the materials performance. Especially, CNF with carboxylated groups can be used as the separation membrane in polymer electrolyte membrane fuel cell. The water dynamics is closely related to the proton conductivity. The Non-destructive quasi-elastic neutron scattering (QENS) is used to characterized water movement in hydrated membrane made of CNF prepared by TEMPO-oxidation with different surface charges. However, neither surface charge nor the nanoconfinement due to membrane swelling has large impact on water dynamics mechanism. A slow diffusive motion is found with the diffusion coefficient close to bulk water and that in hydrated Nafion membrane regardless the surface charge, while a fast motion is rather localized with a correlation time increasing as temperature increase, which might related to the hydrogen bond network formation between water and CNF.
\end{abstract}

\footnotetext{
† Present address: RISE Bioeconomy, Box 5604, 11486 Stockholm, Sweden

* Present address: Dept. of Engineering Sciences, Uppsala University, Box 534, 75121 Uppsala, Sweden
} 


\section{Introduction:}

The water - cellulose interaction is one of the most important scientific topics in the field of woodbased material science. It does not only conceal the secret behind the plant growth, but also determines processing parameters and performance of the final wood-based materials. In nature, cellulose polymer chains self-assemble into nanofibrils which consist of alternative crystalline and non-crystalline region along the fibril long axis. These so-called cellulose nanofibrils (CNF) serve as basic building block and are further organized with other biopolymer in water media to form the plant cell wall.' Compared to many wood-based materials, CNF to the largest extent keep the strong nature of cellulose materials, i.e. preserving excellent mechanical properties ${ }^{23}$. Meanwhile, the high surface-to-volume ratio enables a large surface area per unit weight and the extensive hydroxyl group exposed on the CNF surface could be easily modified and functionalized ${ }^{4 s}$. Especially, the carboxylation of CNF by the TEMPO-oxidation process has now become a common step for most of application as it delivers fine cross-section and activated carboxylated surface ligands ${ }^{6}$. Additionally, CNF are readily extractable from the pulp fibres and biodegradable. Thus, they have become a popular material platform for developing novel composites $^{45,-9 \rightarrow}$. Among many applications, the good mechanical property of CNF has attracted most of the attention for engineering materials. Moisture content in CNF based materials influence both stiffness and strength ${ }^{10}$. On top of the robustness, the CNF could be used alone and/or as a carrier to load other functional materials for versatile purposes in optics" electronics $^{12}$ and energy device ${ }^{13}$, where moisture content and electrolyte are closely relavent. Recently, CNF membranes with surface functional group have been successfully tested as the separation membrane in Polymer electrolyte membrane fuel cells $\mathbf{s}^{1.15}$. The stable ion conductivity at elevated temperature at high moisture content, as well as the low environmental impact, make CNF a great candidate to replace the costly and environmentally expensive Nafion membrane. Apart from the numerous applications, CNF based materials has demonstrated rich

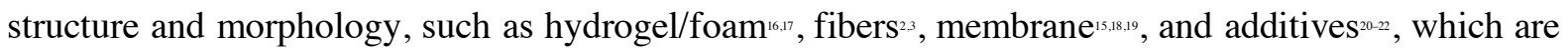
usually in hierarchical structure.

Correspondingly, the water - cellulose interaction also evolves at different scales. At atomic scale, the crystalline structure of CNF is maintained by vast number of hydrogen bond at inter-and intro-CNFs. 
Water could certainly interact with CNF via hydrogen bond. As most of the biopolymer ${ }^{3 s}$ (protein, lipid, and DNA), which usually contains both hydrophilic and hydrophobic part, CNF crystalline phase are also terminated with hydrophilic and hydrophobic surface. Water could hence adsorb on different surface via different mechanism ${ }^{24}$. At nanoscale, $\mathrm{CNF}$ consists of crystalline phase and non-crystalline phase. The crystalline phase may deform upon water uptake ${ }^{2 s}$, while non-crystalline phase are looser and permeable for water ${ }^{*}$. Meanwhile, between CNFs, spatial confinement is also formed. Numbers of work has shown that water in confined space show distinct behaviour compared to bulk water ${ }^{22-30}$. At micron scale, the capillary force on the water in the different CNF superstructure should be taken in account, just as the cell structure and lumen of wood cell. Despite the multiscale mechanism, water in cellulose matrix can always be classified as "free" water as capillary water in lumen" and "bound" water, which could be further grouped as "immobile" and "mobile" water". Knowing the water dynamics in CNF based material will certainly contribute the material design and eventually benefit the bioeconomy.

Previously, the water - cellulose interaction in cellulose fibres has been studied by NMR spectroscopy, ${ }^{33}$ showing a moisture-content-dependent diffusion coefficient for both fast and slow water motion. IR spectroscopy has been used to study the water adsorption on cellulose fiber surface via hydrogen bond ${ }^{3+3 s}$. Neutron scattering is a non-destructive characterization method to study the water dynamics, which complementary to NMR and IR by providing momentum transfer information thus covering multi spatial $(\AA \sim \mathrm{nm})$ and temporal scale (ps $\sim \mathrm{ns})$. Recently, O’Neill et al. have used QENS to study the dynamics of water bound to crystalline cellulose by use high resolution instrument BASIS and bacterial cellulose with large crystalline size ${ }^{\mathrm{s}}$. Two type of water was reported with different dynamic properties. However, most of published work were carried out on large fibres in microscale. The water dynamics in CNF system is still scarcely reported despite the vast applications. Thus, in this work, we choose to study the water dynamics in the hydrated membrane made of carboxylated CNF prepared via TEMPO oxidation, which a widely used method in CNF preparation and show great reproducibility. The influence of the number of carboxylated groups has been investigated. Thus, the study could serve as a model case of the water dynamics in CNF system. Furthermore, as the similar CNF membranes has been successfully used a separation membrane in fuel cell ${ }^{1+1,5}$, the study could help us understand the 
proton conduction mechanism in $\mathrm{CNF}$ and develop more efficient and environment-friendly energy device.

\section{Materials and Experimental Methods:}

Carboxylated CNFs with surface charge around $600 \mu \mathrm{eq} / \mathrm{g}$ and $1500 \mu \mathrm{eq} / \mathrm{g}$ were prepared via TEMPOmediated oxidation based on the procedure of Saito et al ${ }^{36} \mathrm{CNF}$ membranes were firstly prepared via solution casting in plastic Petri dishes with a diameter of $5.5 \mathrm{~cm}$ in controlled relative humidity (RH) of $50 \%$ at $30{ }^{\circ} \mathrm{C}$ for 48 hours. Then, both CNF600 and CNF1500 membrane were reconditioned at RH $90 \%$ at $30{ }^{\circ} \mathrm{C}$ over 24 hours for high moisture content. These samples are named as CNF600 and CNF1500, respectively. The reconditioned membranes were stacked together and sealed in plate-like aluminium sample holder via In-Sn alloy wire as gasket. In addition, some of CNF600 membranes were conditioned at $\mathrm{RH} 10 \%$ at $30{ }^{\circ} \mathrm{C}$ and sealed in the aluminium sample holder as a reference sample, named as CNF600 Dry, while some CNF1500 were conditioned at RH 50\% to check the water content influence.

QENS measurements were performed at IRIS Time-of-flight (TOF) inverted-geometry crystal analyser spectrometer, ISIS neutron facility, UK. 51 sets of ZnS scintillator detectors were binned down to 17 groups, covering the range of transferred wavevector (Q) from 0.5 to $1.9 \AA$. The QENS spectrum at each $\mathrm{Q}$ were converted to the format with the energy range of $+/-0.5 \mathrm{meV}$ and re-binned energy step of $0.002 \mathrm{meV}$ for analyses. Vanadium was measured for resolution calibration and data reduction. QENS of CNF600 measured at $20 \mathrm{~K}$ was used as resolution function for further QENS analyses of other CNF specimen at higher temperatures. The data reduction and Bayesian-spectral-analyses fitting were carried out via analysis software Mantid ${ }^{\natural}$. The fitting component uses Lorentzian functions for quasielastic peaks while taking slope background and $\delta$-function for elastic line. In general, a QENS spectrum can be modelled by the following equation:

$$
I(Q, E)=\left[A(Q) \cdot \delta(Q, E)+(1-A(Q)) \cdot \sum_{n=1}^{N} B_{n} \cdot L_{n}\left(Q, \Gamma_{n}\right)\right] \otimes R(Q, E)+C(Q, E)
$$


where $\mathrm{A}(\mathrm{Q})$ is the ratio of elastic scattering component; $\delta(Q, E)$, delta-function, depicts the elastic scattering without energy transfer. $B_{n}$ is the contribution factor of different quasi-elastic peak, which is described by single or multiple Lorentzian function $L(Q, \Gamma)$, with $\Gamma$ as the full-width at half maximum (FWHM). Both elastic and quasi-elastic peak should be convoluted with resolution function $R(Q, E)$ by considering the experimental resolution. $C(Q, E)$ is the background.

\section{Results and Discussions:}

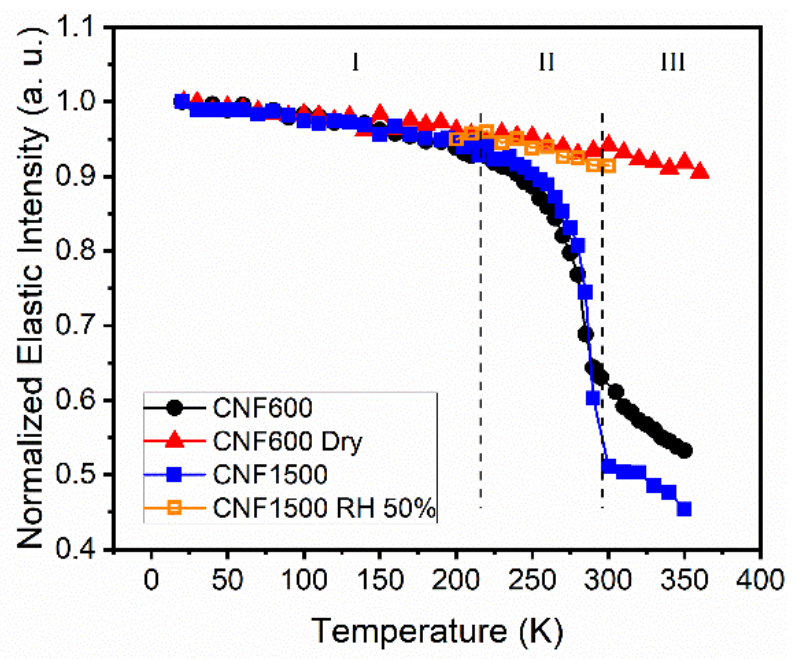

Figure 1. Elastic window scan, i.e. the elastic peak intensity of QENS as a function of temperature of CNF600

(black), CNF1500 (blue), CNF600 Dry (red) and CNF1500 RH 50\% (yellow). Peak intensities at different temperatures are integrated around $\mathrm{Q} \sim 0.77 \AA$ and within the energy range of $\pm 0.0175 \mathrm{meV}$, and are normalized to the one at $20 \mathrm{~K}$.

The water dynamics in hydrated CNF membrane analyses started with determining the elastic intensity evolution as a function of temperature. At very low temperature the decrease in elastic intensity is due to vibrations which are according to the temperature factor more and more excited. The intensity decrease follows the Debye-Waller factor. The trend is shown in Figure 1. In general, as temperature increase, a further elastic intensity decrease indicates particle movements beyond harmonic vibrations These are stochastic movements of translational and rotational character and will appear as quasi-elastic neutron scattering signal. Clearly, CNF600 and CNF1500 show a very distinct three stage (I - III) diagram. However, for CNF600 Dry, it only shows a slowly linear decrease to the extent of 5\%. Such 
difference between hydrated and low water content CNF suggests that the water movement in the membrane result in dramatic intensity drop for hydrated membranes. We also investigated water content influence by reconditioning some CNF1500 at RH 50\% and did the elastic windows scan between 200 and $310 \mathrm{~K}$. The initial of value is normalized a random value between those of CNF600 Dry and CNF1500 for intermediate water content. It is found that at water uptake in the CNF membrane at RH 50\% show rather similar trend as CNF600 Dry, meaning that water movement is not sufficiently strong in the system. Given the extensive surface area in CNF membrane, water taken up at RH 50\% may still bound to the membrane surface but generate enough diffusion movement to elaborate the quasi-elastic contribution. There has been a structural hint for this phenomenon in our recently work's in which small angle X-ray scattering (SAXS) results show that the membrane structures did not change below RH $70 \%$, suggesting that water are rather evenly distributed on the membrane surfaces but create any structure change. Regarding this three-stage diagram, all systems show a similar trend in Stage I below $200 \mathrm{~K}$, meaning all water molecules are frozen and/or bound firmly. Stage II is a transition stage where some water molecules has gained enough thermal activation energy become mobile. The development become much steep around $290 \mathrm{~K}$ and become slowed down afterwards where a different mechanism is believed to dominate.

To quantitatively evaluate the water dynamics mechanisms, QENS spectrum require curve deconvolution by using Equation (1). However, in many cases, the discrete summation of the quasielastic peaks is not sufficiently straightforward to model the spectrum, one has to convert QENS into intermediate scattering function in time-domain via Fourier transformation and fit the results with stretched exponential ${ }^{\infty s i 9}$. One way to circumvent the difficulties and identify the contribution of the number of Lorentzian component is to calculate the dynamics susceptibility $\chi^{\prime \prime}(Q, E)$ by normalizing the QENS spectrum to Bose population factor $n_{s}$ (Eq. 2).

$$
\chi^{\prime \prime}(Q, E)=\frac{I(Q, E)}{n_{B}(T, E)}
$$

Where, $n_{B}(T, E)=\left(1-\exp \left(-E / k_{B} T\right)\right)^{-1}, \mathrm{E}$ is the transferred energy, $\mathrm{T}$ is the temperature and $\mathrm{k}_{\mathrm{B}}$ is the Boltzmann constant ${ }^{*}$. 

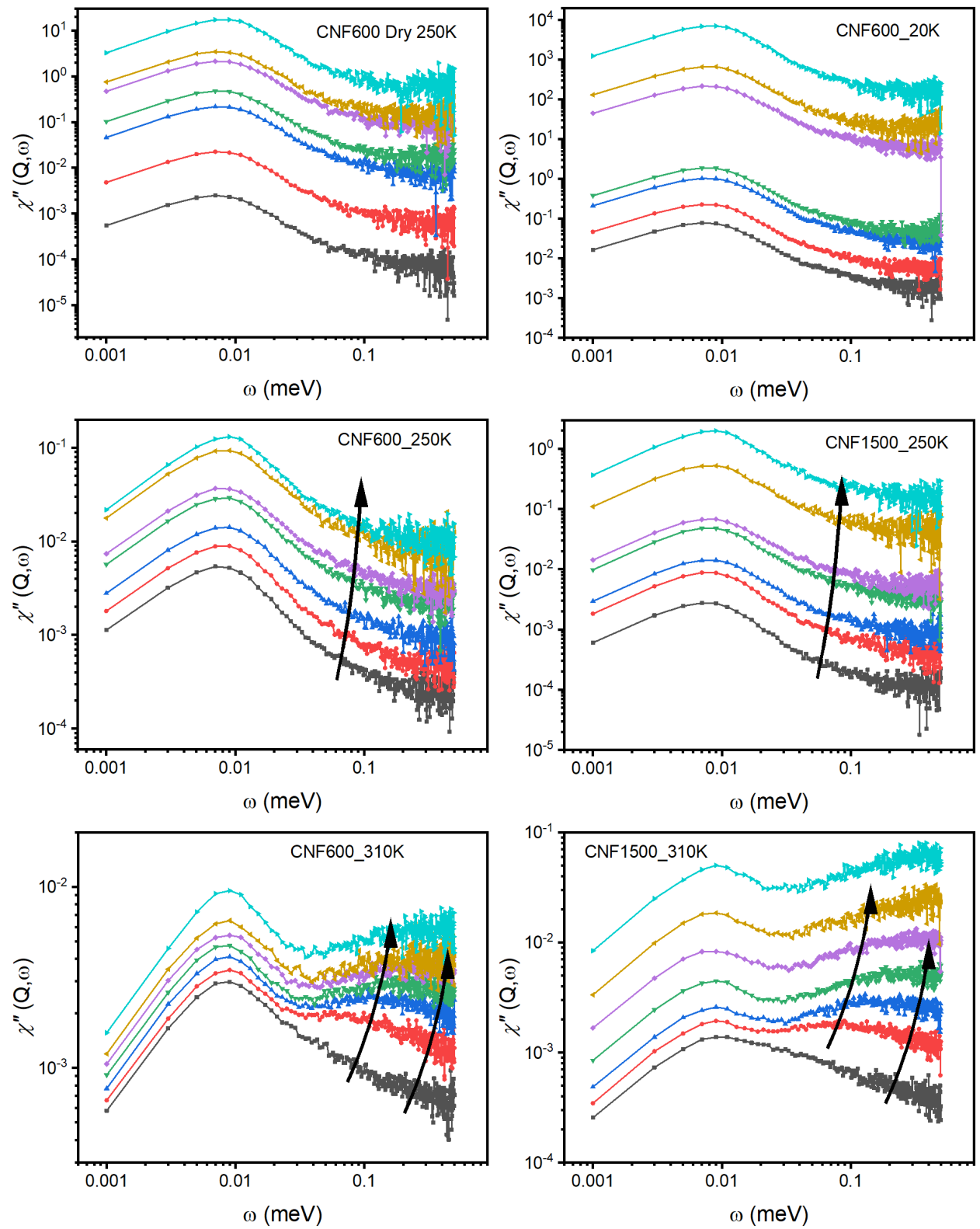

Figure 2. dynamic susceptibility of CNF600 and CNF1500 at $250 \mathrm{~K}$ and 310K for 7 selected transferred wavevector $Q$ at $0.48 \AA^{\prime}$ (grey), $0.84 \AA^{4^{\prime}}$ (red), $1.17 \AA^{-1}$ (blue), $1.54 \AA^{-1}$ (green), $1.60 \AA^{\prime}$ (purple), $1.72 \AA^{\prime}$ (yellow), and $1.84 \AA$ (cyan). The spectra were shifted vertical to highlight the dispersity. Solid Arrow are guides to show the possible components at $250 \mathrm{~K}$.

Dynamic susceptibility excludes the contribution of elastic term and easily unveil different dynamic process $^{3.1+1+2 .}$. Figure 2 shows dynamic susceptibility of CNF600 and CNF1500 at selected temperature and water content (full set of dynamic susceptibility is available in support information). For both CNF600 Dry and CNF600 at $20 \mathrm{~K}$, the water molecules are either too few or immobile. Their dynamic 
susceptibility also shows similar structure will a dominant peak around $0.01 \mathrm{meV}$, which is also independent of Q (i.e. non-dispersive).

Such features have been found for all specimen at different temperature, thus a systematic result which might be related to $\mathrm{CNF}$ membrane inherent dynamics or experimental setup. When temperature increase till $250 \mathrm{~K}$, both CNF600 and CNF1500 shows increase of $\chi^{\prime \prime}(Q, E)$ at higher transferred energy around $0.05 \mathrm{meV}$, which also shows slight dispersive relation as a function of Q. At even higher transfer energy, it becomes a bit ambiguous if another dispersive dynamic channel exists at $250 \mathrm{~K}$. However, at 310K, two dispersive dynamic channels become clearly visible.
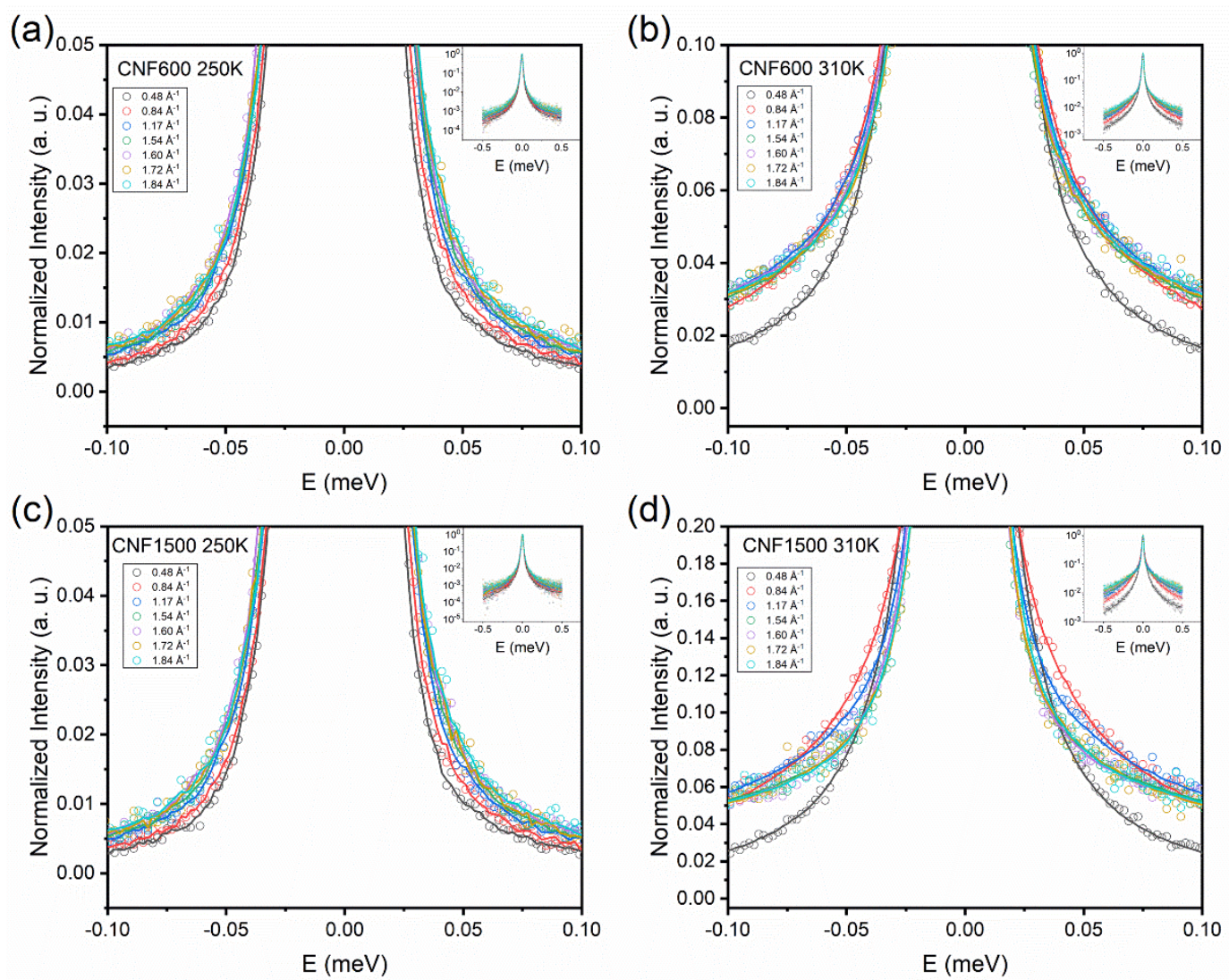

Figure 3. Zoomed QENS and Bayesian fitting with two-components Lorentzian functions for CNF600 and CNF1500 at $250 \mathrm{~K}$ and $310 \mathrm{~K}$. Open circles are experimental data and solid lines are fitting results. Bayesian analyses was carried out by software Mantid ${ }^{3}$. Inserts show the data in full scale.

The result of the dynamic susceptibility indicates that two-component Lorentzian functions are plausible for quasi-elastic scattering, at least at higher temperature in stage III. Selected fitting results 
are summarized in Figure 3. (The full sets of QENS spectra are available in support information). To highlight the broadening of QENS peak, all the data are normalized to peak maximum. As Q increase, the QENS peaks become broadened, in line with the dispersity shown by dynamic susceptibility. In combination with Bayesian analyses, two-components Lorentzian model indeed provides reasonably good fit (solid color-coded line in Figure 3.). Such two components are fitted with distinct widths which are correspondent to slow and fast motions.

The Half-width at half maximum (HWHM, or $\Gamma / 2)$ of Lorentzian function of the slow motion are extracted and plotted in Figure 4. The dispersive relation of HWHM as a function of Q reveals a diffusive process. Typically, jump diffusion is a common mechanism in nanoscale, where molecule could only hop between different site instead of continuously moving as in the macroscopic scale. However, there are several types of jump diffusion model, e.g. Chudley-Elloitt (CE) model ${ }^{\text {s }}$, SingwiSjölander (SS) model ${ }^{4}$ and Hall-Ross (HR) model (Eq. 2$)^{4 s}$.

The CE model assumes that the particle jumps only a certain distance 1, which is a severe constraint in particular for a disordered system. To overcome this constraint the SS model assumes an exponential distribution of jump lengths and the HR model assumes a Gaussian distribution of jump lengths around a mean value.

CE model depicts the jump on lattice; SS model describes the jump decay as an exponential function; while HR model describes the jump in Gaussian-type confined space. The slow motion HWHM were tentatively fitted by all three models. (see support information for details). Chi-square analysis suggests that HR model (Eq. 3) gives the relatively better fitting which are summarized and plotted in Figure 4 (a) and (b).

$$
\frac{\Gamma}{2}=\frac{\hbar}{\tau}\left(1-\exp \left(-\frac{Q^{2} l^{2}}{6}\right)\right)
$$

where, $\hbar$ is reduced Plank constant and $l$ is the mean jump length and $(\tau)$ denotes the residence time between consecutive jumps. Note that the $250 \mathrm{~K}$ results are near the energy resolution of the spectrometer. As temperature increases, the increase of HWHM is translated as a decreased residence 
time and longer mean jump diffusion length (see Table 1), indicating the motion becomes faster. Based on the fitting parameters obtained in Eq.3, the diffusion coefficient $(D)$ is calculated via Eq. 4.

$$
D=\frac{l^{2}}{6 \tau}
$$

In Figure 4 (c), the diffusion coefficient becomes larger as the temperature increases. For both CNF600 and CNF1500, their D values are rather close despite their different numbers of carboxylated ligands on fibrills' surface. The D values at $310 \mathrm{~K}$ are around $2 \times 10^{\mathrm{s}} \mathrm{cm}^{2} / \mathrm{s}$, which is very close to the bulk water diffusion coefficient ${ }^{\star \Delta+17}$ and that in hydrated Nafion ${ }^{\star s}$ and perfluorinated sulfonic acid (PFSA) $)^{* 2}$ membranes. Thus, this slow motion is probably related to the bulk-like water in the membranes. This fact supports the application of CNF as separation membrane in fuel cells. ${ }^{1.15}$ On the other hands, the D values at $250 \mathrm{~K}$ and $270 \mathrm{~K}$ are 2 times higher than those determined values in crystalline cellulose ${ }^{\text {s"}}$, indicating that nanofibril networks may have less restriction to the water movement compared to the crystalline phase.

Table 1. Fitting parameters of Hall-Ross jump diffusion model in Figure 4.

\begin{tabular}{ccccc}
\hline \hline & $\mathrm{T}(\mathrm{K})$ & $\tau(\mathrm{ps})$ & $1(\AA)$ & $\mathrm{D}\left(10^{-5} \mathrm{~cm}^{2} / \mathrm{s}\right)$ \\
\hline \multirow{4}{*}{ CNF600 } & 250 & $48.3 \pm 2.0$ & $3.3 \pm 0.3$ & $0.4 \pm 0.1$ \\
& 270 & $32.7 \pm 1.5$ & $3.4 \pm 0.4$ & $0.6 \pm 0.1$ \\
& 280 & $28.0 \pm 1.0$ & $4.2 \pm 0.4$ & $1.1 \pm 0.2$ \\
& 310 & $24.3 \pm 0.6$ & $5.0 \pm 0.3$ & $1.7 \pm 0.2$ \\
\hline \multirow{4}{*}{ CNF1500 } & 250 & $39.0 \pm 3.9$ & $2.3 \pm 0.4$ & $0.2 \pm 0.1$ \\
& 270 & $29.2 \pm 1.6$ & $3.5 \pm 0.4$ & $0.7 \pm 0.2$ \\
& 280 & $24.4 \pm 1.3$ & $3.9 \pm 0.4$ & $1.0 \pm 0.2$ \\
\hline \hline
\end{tabular}

Note that the hydration of CNF membranes at $\mathrm{RH} 95 \%$ generates more nanostructures than those conditioned at RH 55\%, which is related to the swelling of the membrane (see Figure 4 (d)). The SAXS data could be reasonably fitted by a linear combination of a power-law function and a Gaussian function.

$$
I(q)=\frac{A}{Q^{n}}+B \cdot \exp \left(-\frac{Q^{2} \cdot \Xi^{2}}{2}\right)+C
$$


Where, $\mathrm{A}$ and $\mathrm{B}$ are scalling factor, and $\mathrm{C}$ is background. The power-law function depicts the network connection by the exponent (n) in the low Q region $\left(<0.03 \AA^{-}\right)$. In the case of RH 95\%, CNF600 and CNF1500 have the exponent value around 1.3 and 1.8, respectively, compared to that of 2.6 for CNF1500 at RH 55\%. This suggests that fibril network turns from massively connected network (known as mass fractal structure) into less interconnected structure with branched fibre shape dominant. At high Q region $\left(0.03 \AA^{-1}<\mathrm{Q}<0.2 \AA^{-1}\right)$, Gaussian functions reasonably reproduce the features due to intensity increase at RH 95\%, which are generally assigned to entangled nanostructure with the radiusof-gyration $(\Xi)$. However, $\Xi$ are found around $1.5 \mathrm{~nm}$ in both CNFs and are much larger than the mean jump length $l$ of $3 \sim 5 \AA$ determined from QENS (Table 1). So, the slow motion is still governed by even smaller scale features and the size of nanopores in the membrane might not have direct influence on the water dynamics.

Nevertheless, it is known that the difference in the surface charge can result significant difference on water uptake: in this case, the water content in CNF 1500 is around $79.5 \mathrm{wt} \%$ and that of CNF600 is only $34.0 \mathrm{wt} \%$, in this work. Clearly, given the same dry weight content, CNF1500 may contain more bulk-like water (Also the diffusion coefficient at $310 \mathrm{~K}$ is larger). Consequently, the CNF1500 could transport more protons than CNF600 per unit weight. This is also reflected in Figure 1 as that the decreasing trend of elastic intensity is similar for both CNF1500 and CNF600, but CNF1500 reached lower value in the Stage III since more mobile waters are in CNF1500. The result coincides with the proton conductivity measurement of similar kind of CNF membrane in fuel cell deviceis. 


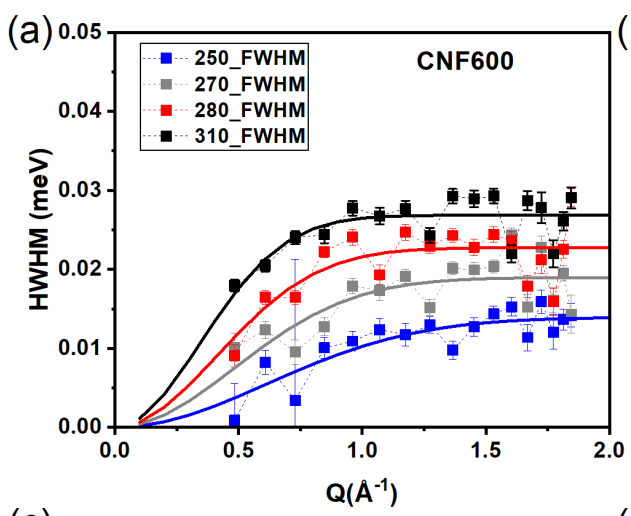

(c)

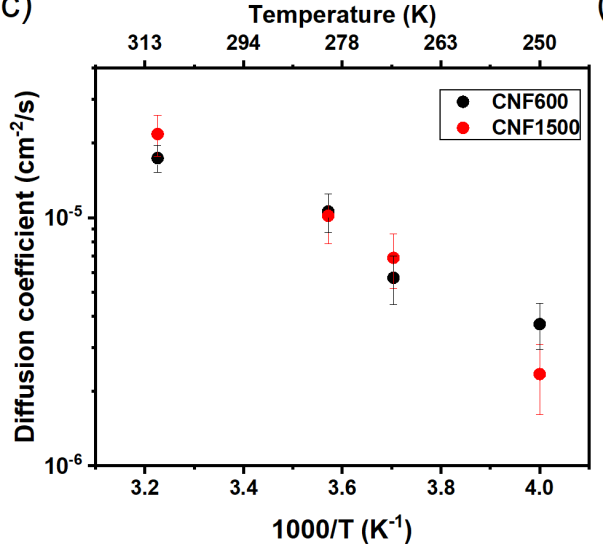

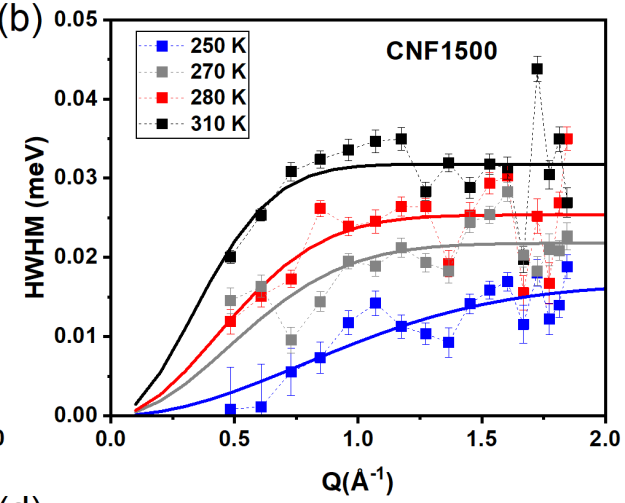

(d)

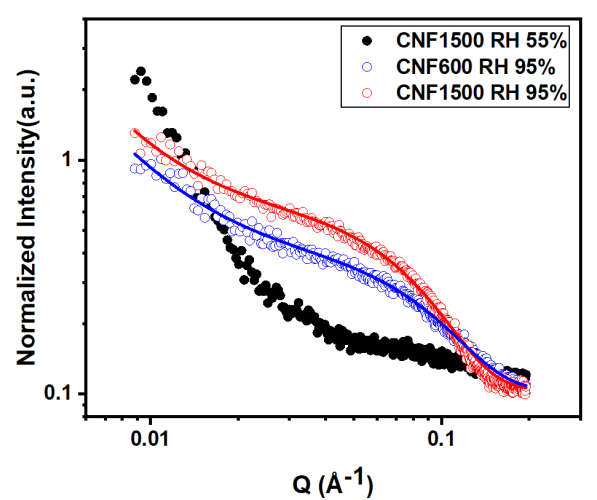

Figure 4. HWHM of the slow motion as a function of transfer wavevector Q and the corresponding fitting results by Hall-Ross jump diffusion model for CNF600 (a) and CNF1500(b). (c) Diffusion coefficient of slow motion. (d) SAXS of CNF600 and CNF1500 at RH 95\% and CNF1500 at RH 55\% as reference. 
(a)
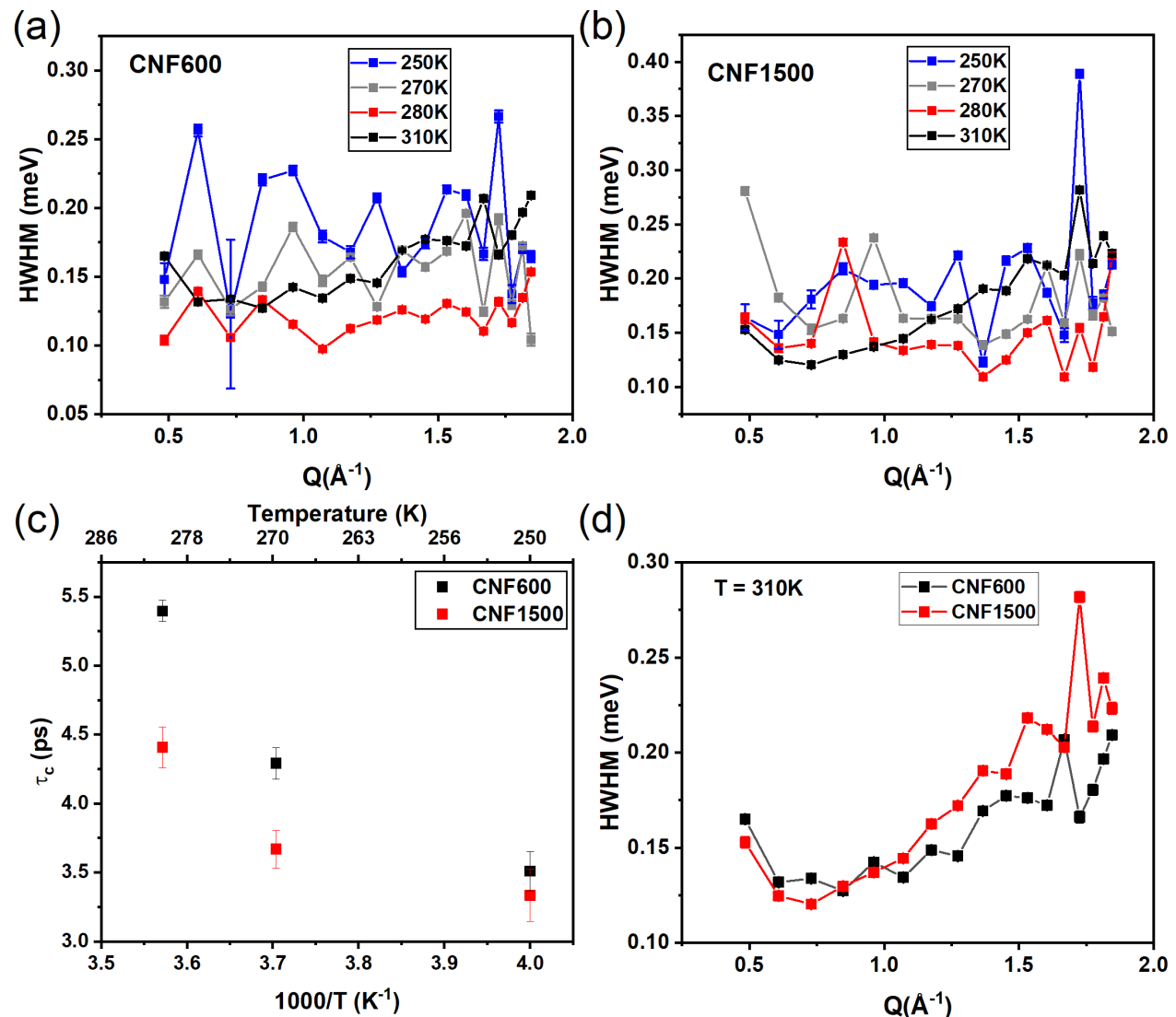

(d)

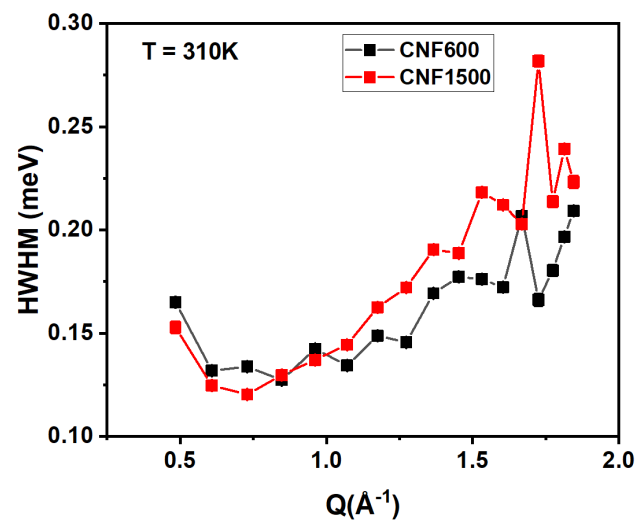

Figure 5. HWHM of the fast motion as a function of transfer wavevector Q for CNF600 (a) and CNF 1500 (b). (c): the correlation time as a function of inversed temperature for CNF600 and CNF1500 at 250, 270 and $280 \mathrm{~K}$. (d): Q-dependent HWHM of the fast motions of CNF600 and CNF1500 at 310K

For the fast motion, their HWHMs are plotted in Figure 5. In both panels of (a) and (b), HWHMs show independence on transfer wavevector $\mathrm{Q}$ from $250 \mathrm{~K}$ to $280 \mathrm{~K}$. This means the fast motion is rather localized and related to bound water. Similar phenomena have been found for water in perfluorinated sulfonic acid (PFSA) materials ${ }^{42}$, SBA-15 mesoporous silica ${ }^{49}$ and graphene oxide ${ }^{s^{\circ}}$. The bound water motion could be converted into correlation time $\left(\tau_{c}\right)$ via $\tau_{c}=2 \hbar / \Gamma_{\text {mean }}$, where $\Gamma_{\text {man }} / 2$ is the mean value of HWHM. The relation between the correlation time and temperature is plotted in Figure 4 (c). Contrary to the residence time of the slow motion, which decreases as the temperature increase, $\tau_{c}$ increases non-linearly as the temperatures increase. Overall, CNF1500 shows smaller $\tau_{\mathrm{c}}$ than CNF600, meaning a faster motion. By combining NMR and analyses approach proposed by Bloch, Purcell and Pound ${ }^{51}$, Mallamace et al.,"si have reported a similar trend of correlation time in nano-second regime 
associated to local Brownian motion in bulk and emulsified water. Such behaviour of $\tau_{\mathrm{c}}$ is related to water structure change from a large disorder phase at higher temperature to a hydrogen-bond-driven structured network below a cross-over temperature $(\sim 290 \mathrm{~K})^{s_{2}}$, which is also close to the transition temperature between Stage II and III in our case (Figure 1). The hydroxyal and carboxylated surface ligand of CNF are known to readily interact with water via hydrogen bond. ${ }^{35}$ Such interaction at surface and confined space may contribute to the anomalous behaviors ${ }^{30}$. At $310 \mathrm{~K}$, the HWHM show a positive Q-dependence. One should be cautious about the upturning of HWHM around $0.5 \AA^{-1}$ for less statistic point, nevertheless a plateau between 0.5 and $1.0 \AA^{*}$ is rather plausible for both CNF600 and CNF1500. Such plateau indicated a dynamic process is in a confined space, which is related to the cage or cagelike structure in the system. Similar phenomena has been reported for ionic liquid systems ${ }^{3: s+4}$.

The cage structure would be related then to the q value when HWHM increase from the plateau. That length scale is definitely much smaller than all scales seen in the SAXS, which brings us back to the 1 value from the diffusion and to what it relates on the atomistic scale.

To briefly summarize the results, QENS show that water dynamics in CNF system could be divided into a three-stage development. Such trend could be related to a diffusive slow motion and a fast localized or confined motion. The slow motion can be well described by Hall-Ross jump diffusion model and the mean jump length is smaller than the size of nanostructure due to membrane swelling. The fast motion shows an abnormal temperature-dependent correlation time, which might be related to hydrogen bond network formation between water and cellulose.

\section{Conclusion:}

We use QENS to investigate the water dynamics in hydrated CNF membranes with carboxylated surface ligands. Such water dynamics is closed related to the proton conductivity, which is especially important for fuel cell application. The determined diffusion coefficient of a slow motion is very close to free water in bulk-like water and those in Nafion membrane, while the fast motion is rather localized or confined given the experimental temperature range. The number of carboxylated groups on CNF surface, similar to the case of water dynamics on charged and un-charged polysaccharide hydrogel ${ }^{54}$. QENS is 
highly sensitive to microscopic mechanism, which may not fully explain the macroscopic properties. The hydrated CNF1500 could uptake nearly as twice much water molecule as CNF600 at RH 95\% and consists of more nanostructure as shown in SAXS. Consequently, the amount of water molecule as proton carriers in CNF1500 are more than CNF600, which could account for proton conductivity difference in real applications.

Acknowledgement: The authors would like to thank Dr. Stewart Parker and Dr. Fanni Juranyi for supports and helpful discussions on this project. ISIS neutron facility is acknowledged for granted experimental time and support. The Wallenberg Wood Science Center and the Swedish research council, VR, are acknowledged for financial support.

Supporting information available: This material is available free of charge via the Internet at http://pubs.acs.org. Scanning electron microscopy image of cross section of CNF600 and CNF1500; The full set of dynamic susceptibility of CNF600 and CNF1500; The fitting result of HWHM by Chudley-Elloitt, Hall-Ross and Singwi-Sjölander models and chi-square value of the fitting.

\section{Reference:}

(1) Uraki, Y.; Koda, K. Utilization of Wood Cell Wall Components. J. Wood Sci. 2015, 61 (5), 447-454. https://doi.org/10.1007/s10086-015-1492-9.

(2) Nechyporchuk, O.; Håkansson, K. M. O.; V, K. G.; Lundell, F.; Hagström, B.; Köhnke, T. Continuous Assembly of Cellulose Nanofibrils and Nanocrystals into Strong Macrofibers through Microfluidic Spinning. 2018, 1800557, 1-10. https://doi.org/10.1002/admt.201800557.

(3) Håkansson, K. M. O. K. M. O.; Fall, A. B. A. B. A. B.; Lundell, F.; Yu, S.; Krywka, C.; Roth, S. V. S. V.; Santoro, G.; Kvick, M.; Prahl Wittberg, L.; Wågberg, L.; et al. Hydrodynamic Alignment and Assembly of Nanofibrils Resulting in Strong Cellulose Filaments. Nat. Commun. 2014, 5 (1), 4018. https://doi.org/10.1038/ncomms5018.

(4) Klemm, D.; Kramer, F.; Moritz, S.; Lindström, T.; Ankerfors, M.; Gray, D.; Dorris, A. 
Nanocelluloses: A New Family of Nature-Based Materials. Angew. Chem. Int.Ed. Engl. 2011, 50 (24), 5438-5466. https://doi.org/10.1002/anie.201001273.

(5) Thomas, B.; Raj, M. C.; B, A. K.; H, R. M.; Joy, J.; Moores, A.; Drisko, G. L.; Sanchez, C. Nanocellulose, a Versatile Green Platform: From Biosources to Materials and Their Applications. Chem. Rev. 2018, acs.chemrev.7b00627. https://doi.org/10.1021/acs.chemrev.7b00627.

(6) Isogai, A.; Saito, T.; Fukuzumi, H. TEMPO-Oxidized Cellulose Nanofibers. Nanoscale 2011, 3 (1), 71-85. https://doi.org/10.1039/C0NR00583E.

(7) Dufresne, A. Nanocellulose: A New Ageless Bionanomaterial. Mater. Today 2013, 16 (6), 220-227. https://doi.org/10.1016/j.mattod.2013.06.004.

(8) De France, K. J.; Hoare, T.; Cranston, E. D. Review of Hydrogels and Aerogels Containing Nanocellulose. Chem. Mater. 2017, 29 (11), 4609-4631. https://doi.org/10.1021/acs.chemmater.7b00531.

(9) Lee, K.-Y.; Aitomäki, Y.; Berglund, L. A.; Oksman, K.; Bismarck, A. On the Use of Nanocellulose as Reinforcement in Polymer Matrix Composites. Compos. Sci. Technol. 2014, 105, 15-27. https://doi.org/10.1016/j.compscitech.2014.08.032.

(10) Lundahl, M. J.; Cunha, A. G.; Rojo, E.; Papageorgiou, A. C.; Rautkari, L.; Arboleda, J. C.; Rojas, O. J. Strength and Water Interactions of Cellulose I Filaments Wet-Spun from Cellulose Nanofibril Hydrogels. Nat. Publ. Gr. 2016, No. February, 1-13. https://doi.org/10.1038/srep30695.

(11) Zhu, H.; Fang, Z.; Preston, C.; Li, Y.; Hu, L. Transparent Paper: Fabrications, Properties, and Device Applications. Energy Environ. Sci. 2014, 7 (1), 269. https://doi.org/10.1039/c3ee43024c.

(12) Tobjörk, D.; Österbacka, R. Paper Electronics. Adv. Mater. 2011, 23 (17), 1935-1961. https://doi.org/10.1002/adma.201004692. 
(13) Wang, X.; Yao, C.; Wang, F.; Li, Z. Cellulose-Based Nanomaterials for Energy Applications. Small 2017, 13 (42), 1-19. https://doi.org/10.1002/smll.201702240.

(14) Bayer, T.; Cunning, B. V.; Selyanchyn, R.; Nishihara, M.; Fujikawa, S.; Sasaki, K.; Lyth, S. M. High Temperature Proton Conduction in Nanocellulose Membranes: Paper Fuel Cells. Chem. Mater. 2016, 28 (13), 4805-4814. https://doi.org/10.1021/acs.chemmater.6b01990.

(15) Guccini, V.; Carlson, A.; Yu, S.; Lindbergh, G.; Wreland Lindström, R.; Salazar-Alvarez, G. Highly Proton Conductive Membranes Based on Carboxylated Cellulose Nanofibres and Their Performance in Proton Exchange Membrane Fuel Cells. ChemRxiv. Prepr. 2019. https://doi.org/10.26434/chemrxiv.7851461.v3.

(16) De France, K. J.; Hoare, T.; Cranston, E. D. Review of Hydrogels and Aerogels Containing Nanocellulose. Chem. Mater. 2017, 29 (11), acs.chemmater.7b00531. https://doi.org/10.1021/acs.chemmater.7b00531.

(17) Chang, C.; Zhang, L. Cellulose-Based Hydrogels: Present Status and Application Prospects. Carbohydr.Polym. 2011, 84 (1), 40-53. https://doi.org/10.1016/j.carbpol.2010.12.023.

(18) Galland, S.; Andersson, R. L.; Salajková, M.; Ström, V.; Olsson, R. T.; Berglund, L. a. Cellulose Nanofibers Decorated with Magnetic Nanoparticles - Synthesis, Structure and Use in Magnetized High Toughness Membranes for a Prototype Loudspeaker. J. Mater. Chem. C 2013, 1 (47), 7963. https://doi.org/10.1039/c3tc31748j.

(19) Voisin, H.; Bergström, L.; Liu, P.; Mathew, A. Nanocellulose-Based Materials for Water Purification. Nanomaterials 2017, 7 (3), 57. https://doi.org/10.3390/nano7030057.

(20) Biyani, M. V; Foster, E. J.; Weder, C. Light-Healable Supramolecular Nanocomposites Based on Modified Cellulose Nanocrystals. ACS Macro Lett. 2013, 2 (3), 236-240. https://doi.org/10.1021/mz400059w.

(21) Tkalya, E.; Ghislandi, M.; Thielemans, W.; van der Schoot, P.; de With, G.; Koning, C. Cellulose Nanowhiskers Templating in Conductive Polymer Nanocomposites Reduces 
Electrical Percolation Threshold 5-Fold. ACS Macro Lett. 2013, 2 (2), 157-163.

https://doi.org/10.1021/mz300597j.

(22) Aydemir, D. Morphological and Thermal Properties of Cellulose Nanofibrils Reinforced Epoxy Nanocomposites. Drv. Ind. 2015, 66 (1), 35-40.

https://doi.org/10.5552/drind.2015.1403.

(23) Biedermannová, L.; Schneider, B. Hydration of Proteins and Nucleic Acids: Advances in Experiment and Theory. A Review. Biochim. Biophys. Acta - Gen. Subj. 2016, 1860 (9), 18211835. https://doi.org/10.1016/j.bbagen.2016.05.036.

(24) Lindman, B.; Karlström, G.; Stigsson, L. On the Mechanism of Dissolution of Cellulose. J. Mol. Liq. 2010, 156 (1), 76-81. https://doi.org/10.1016/j.molliq.2010.04.016.

(25) Zabler, S.; Paris, O.; Burgert, I.; Fratzl, P. Moisture Changes in the Plant Cell Wall Force Cellulose Crystallites to Deform. J. Struct. Biol. 2010, 171 (2), 133-141. https://doi.org/10.1016/j.jsb.2010.04.013.

(26) Müller, M.; Czihak, C.; Schober, H.; Nishiyama, Y.; Vogl, G. All Disordered Regions of Native Cellulose Show Common Low-Frequency Dynamics. Macromolecules 2000, 33 (5), 1834-1840. https://doi.org/10.1021/ma9912271.

(27) Marbach, S.; Dean, D. S.; Bocquet, L. Transport and Dispersion across Wiggling Nanopores. Nat. Phys. 2018, 14 (11), 1108-1113. https://doi.org/10.1038/s41567-018-0239-0.

(28) Briganti, G.; Rogati, G.; Parmentier, A.; Maccarini, M.; De Luca, F. Neutron Scattering Observation of Quasi-Free Rotations of Water Confined in Carbon Nanotubes. Sci. Rep. 2017, 7 (October 2016), 1-10. https://doi.org/10.1038/srep45021.

(29) Kamitakahara, W. A.; Wada, N. Neutron Spectroscopy of Water Dynamics in NaX and NaA Zeolites. Phys. Rev. E - Stat. Nonlinear, Soft Matter Phys. 2008, 77 (4), 1-10. https://doi.org/10.1103/PhysRevE.77.041503.

(30) Biswas, R.; Bagchi, B. Anomalous Water Dynamics at Surfaces and Interfaces: Synergistic 
Effects of Confinement and Surface Interactions. J. Phys. Condens. Matter 2018, 30 (1). https://doi.org/10.1088/1361-648X/aa9b1d.

(31) O’Neill, H.; Pingali, S. V.; Petridis, L.; He, J.; Mamontov, E.; Hong, L.; Urban, V.; Evans, B.; Langan, P.; Smith, J. C.; et al. Dynamics of Water Bound to Crystalline Cellulose. Sci. Rep. 2017, 7 (1), 1-13. https://doi.org/10.1038/s41598-017-12035-w.

(32) Lindh, E. L.; Terenzi, C.; Salmén, L.; Furó, I. Water in Cellulose: Evidence and Identification of Immobile and Mobile Adsorbed Phases by 2H MAS NMR. Phys. Chem. Chem. Phys. 2017, 19 (6), 4360-4369. https://doi.org/10.1039/c6cp08219j.

(33) Perkins, E. L.; Batchelor, W. J. Water Interaction in Paper Cellulose Fibres as Investigated by NMR Pulsed Field Gradient. Carbohydr. Polym. 2012, 87 (1), 361-367. https://doi.org/10.1016/j.carbpol.2011.07.065.

(34) Lindh, E. L.; Salmén, L. Surface Accessibility of Cellulose Fibrils Studied by HydrogenDeuterium Exchange with Water. Cellulose 2017, 24 (1), 21-33. https://doi.org/10.1007/s10570-016-1122-8.

(35) Lindh, E. L.; Bergenstråhle-Wohlert, M.; Terenzi, C.; Salmén, L.; Furó, I. Non-Exchanging Hydroxyl Groups on the Surface of Cellulose Fibrils: The Role of Interaction with Water. Carbohydr.Res. 2016, 434, 136-142. https://doi.org/10.1016/j.carres.2016.09.006.

(36) Saito, T.; Kimura, S.; Nishiyama, Y.; Isogai, A. Cellulose Nanofibers Prepared by TEMPOMediated Oxidation of Native Cellulose. Biomacromolecules 2007, 8 (8), 2485-2491. https://doi.org/10.1021/bm0703970.

(37) Arnold, O.; Bilheux, J. C.; Borreguero, J. M.; Buts, A.; Campbell, S. I.; Chapon, L.; Doucet, M.; Draper, N.; Ferraz Leal, R.; Gigg, M. A.; et al. Mantid-Data Analysis and Visualization Package for Neutron Scattering and $\mu$ SR Experiments. Nucl. Instruments Methods Phys. Res. Sect. A Accel. Spectrometers, Detect. Assoc. Equip. 2014, 764, 156-166. https://doi.org/10.1016/j.nima.2014.07.029. 
(38) Fratini, E.; Chen, S. H.; Baglioni, P.; Cook, J. C.; Copley, J. R. D. Dynamic Scaling of Quasielastic Neutron Scattering Spectra from Interfacial Water. Phys. Rev.E - Stat. Physics, Plasmas, Fluids, Relat. Interdiscip. Top. 2002, 65 (1), 1-4.

https://doi.org/10.1103/PhysRevE.65.010201.

(39) Kikuchi, T.; Nakajima, K.; Ohira-Kawamura, S.; Inamura, Y.; Yamamuro, O.; Kofu, M.;

Kawakita, Y.; Suzuya, K.; Nakamura, M.; Arai, M. Mode-Distribution Analysis of

Quasielastic Neutron Scattering and Application to Liquid Water. Phys. Rev.E 2013, 87 (6), 062314. https://doi.org/10.1103/PhysRevE.87.062314.

(40) Berrod, Q.; Lagrené, K.; Ollivier, J.; Zanotti, J. Inelastic and Quasi-Elastic Neutron Scattering. Application to Soft-Matter. EPJ Web Conf. 2018, 188, 05001. https://doi.org/10.1051/epjconf/201818805001.

(41) Nickels, J. D.; O’Neill, H.; Hong, L.; Tyagi, M.; Ehlers, G.; Weiss, K. L.; Zhang, Q.; Yi, Z.; Mamontov, E.; Smith, J. C.; et al. Dynamics of Protein and Its Hydration Water: Neutron Scattering Studies on Fully Deuterated GFP. Biophys. J. 2012, 103 (7), 1566-1575. https://doi.org/10.1016/j.bpj.2012.08.046.

(42) Berrod, Q.; Lyonnard, S.; Guillermo, A.; Ollivier, J.; Frick, B.; Gébel, G. QENS Investigation of Proton Confined Motions in Hydrated Perfluorinated Sulfonic Membranes and SelfAssembled Surfactants. EPJ Web Conf. 2015, 83, 02002. https://doi.org/10.1051/epjconf/20158302002.

(43) Chudley, C. T.; Elliott, R. J. Neutron Scattering from a Liquid on a Jump Diffusion Model. Proc. Phys. Soc. 1961, 77 (2), 353-361. https://doi.org/10.1088/0370-1328/77/2/319.

(44) Singwi, K. S.; Sjölander, A. Resonance Absorption of Nuclear Gamma Rays and the Dynamics of Atomic Motions. Phys. Rev. 1960, 120 (4), 1093-1102. https://doi.org/10.1103/PhysRev.120.1093.

(45) Hall, P. L.; Ross, D. K. Incoherent Neutron Scattering Functions for Random Jump Diffusion 
in Bounded and Infinite Media. Mol. Phys. 1981, 42 (3), 673-682.

https://doi.org/10.1080/00268978100100521.

(46) González Sánchez, F.; Jurányi, F.; Gimmi, T.; Van Loon, L.; Unruh, T.; Diamond, L. W. Translational Diffusion of Water and Its Dependence on Temperature in Charged and Uncharged Clays: A Neutron Scattering Study. J. Chem. Phys. 2008, 129 (17). https://doi.org/10.1063/1.3000638.

(47) Amann-Winkel, K.; Bellissent-Funel, M.-C.; Bove, L. E.; Loerting, T.; Nilsson, A.; Paciaroni, A.; Schlesinger, D.; Skinner, L. X-Ray and Neutron Scattering of Water. Chem. Rev. 2016, 116 (13), 7570-7589. https://doi.org/10.1021/acs.chemrev.5b00663.

(48) Perrin, J. C.; Lyonnard, S.; Volino, F. Quasielastic Neutron Scattering Study of Water Dynamics in Hydrated Nafion Membranes. J. Phys. Chem. C 2007, 111 (8), 3393-3404. https://doi.org/10.1021/jp065039q.

(49) Kiwilsza, A.; Pajzderska, A.; Gonzalez, M. A.; Mielcarek, J.; Wąsicki, J. QENS and NMR Study of Water Dynamics in SBA-15 with a Low Water Content. J. Phys. Chem. C 2015, 119 (29), 16578-16586. https://doi.org/10.1021/acs.jpcc.5b02672.

(50) Buchsteiner, A.; Lerf, A.; Pieper, J. Water Dynamics in Graphite Oxide Investigated with Neutron Scattering. J. Phys. Chem. B 2006, 110 (45), 22328-22338. https://doi.org/10.1021/jp0641132.

(51) Mallamace, F.; Corsaro, C.; Mallamace, D.; Vasi, S.; Stanley, H. E. NMR Spectroscopy Study of Local Correlations in Water. J. Chem. Phys. 2016, 145 (21). https://doi.org/10.1063/1.4968589.

(52) Xu, L.; Mallamace, F.; Yan, Z.; Starr, F. W.; Buldyrev, S. V.; Eugene Stanley, H. Appearance of a Fractional Stokes-Einstein Relation in Water and a Structural Interpretation Ofits Onset. Nat. Phys. 2009, 5 (8), 565-569. https://doi.org/10.1038/nphys1328.

(53) Embs, J. P.; Burankova, T.; Reichert, E.; Hempelmann, R. Cation Dynamics in the Pyridinium 
Based Ionic Liquid 1- N -Butylpyridinium Bis((Trifluoromethyl)Sulfonyl) as Seen by Quasielastic Neutron Scattering. J. Phys. Chem. B 2012, 116 (44), 13265-13271. https://doi.org/10.1021/jp3070344.

(54) Burankova, T.; Hempelmann, R.; Wildes, A.; Embs, J. P. Collective Ion Diffusion and Localized Single Particle Dynamics in Pyridinium-Based Ionic Liquids. J. Phys. Chem. B 2014, 118 (49), 14452-14460. https://doi.org/10.1021/jp5092416. 